\title{
Influence des opérations thermiques impliquées dans les procédés traditionnels d'extraction du beurre de karité au Bénin
}

\author{
Clément AHOUANNOU ${ }^{1}$, Fidèle P. TCHOBO ${ }^{2}$, Chakirou A. TOUKOUROU ${ }^{1}$, \\ Fernando KOUGBADI ${ }^{2}$ et Mohamed M. SOUMANOU ${ }^{2 *}$ \\ ${ }^{1}$ Laboratoire d'Energétique et de Mécanique Appliquées (LEMA), Ecole Polytechnique d'Abomey-Calavi, \\ Université d'Abomey-Calavi, 01BP : 2009, Cotonou, Bénin. \\ ${ }^{2}$ Unité de Recherche en Génie Enzymatique, Laboratoire d'Etude et de Recherche en Chimie Appliquée \\ (LERCA), Ecole Polytechnique d'Abomey-Calavi, Université d'Abomey-Calavi, 01BP : 2009 Cotonou, Bénin. \\ *Auteur correspondant ; E-mail :msoumanoufr@yahoo.fr, mohamed.soumanou@epac.uac.bj
}

\section{RESUME}

La qualité du beurre de karité dépend des processus qui ont conduit à son obtention. Dans la présente étude, sont exposés les résultats de l'influence des opérations thermiques impliquées dans les procédés traditionnels d'extraction du beurre de karité (Vitellaria paradoxa) au Bénin. L'effet de l'interaction des opérations thermiques (de cuisson, de séchage, de torréfaction, de friture, de fumage et de chauffage) appliquées au cours de différents procédés d'extraction (Lokpa et Peulh au Bénin, Tchabal au Cameroun) a été évalué. Différents échantillons de beurre ont été obtenus par la méthode Soxhlet à partir des fruits, des noix cuites, des noix cuites séchées, des amandes séchées, des amandes séchées et torréfiées, des amandes séchées et frites, des amandes fumées. Les indices d'acide, de peroxyde et de réfraction des différents échantillons de beurre ont été déterminés; de même que la teneur en eau et la composition en acide gras. Les résultats obtenus ont montré que les opérations thermiques permettent la réduction de l'acidité des beurres. Quant au taux de peroxyde, il diminue avec des températures supérieures à $145^{\circ} \mathrm{C}$. Par ailleurs, les multiples résultats obtenus montrent que la chaleur n'a pas d'effet sur la composition en acides gras du beurre.

(c) 2013 International Formulae Group. All rights reserved.

Mots clés : Karité, procédés traditionnels, opérations thermiques, beurre, qualité.

\section{INTRODUCTION}

Le karité, Vitellaria paradoxa, est un arbre tropical d'Afrique de la famille des Sapotacées, typique des savanes arborées soudaniennes, surtout connu par le beurre extrait de ses amandes, d'où l'appellation d'arbre à beurre. Cet arbre à usages multiples joue un rôle socio-économique important dans les communautés rurales en Afrique subsaharienne (Compaoré, 2000). L'arbre de karité donne de grosses baies charnues renfermant, le plus souvent, une à deux ou trois noix à coques fines et cassantes. Cette coque est remplie d'une amande composée de deux cotylédons dont on extrait le principal produit du karité : le beurre. Le beurre de karité est traditionnellement utilisé comme source d'huile végétale pour l'alimentation et les soins corporels. Il est également utilisé en chocolaterie, en pharmacie et en cosmétologie pour ses propriétés intrinsèques liées à sa 
composition en glycérides et sa teneur élevée en insaponifiable (Kapseu et al., 2001).

$\mathrm{Au}$ Bénin, la transformation des amandes du karité en beurre se fait essentiellement par des méthodes traditionnelles et demeure une activité principalement féminine. L'émergence de marchés internationaux pour ce produit offre une opportunité aux femmes rurales qui ont accès à peu d'activités rémunératrices. Sur ces marchés, les exigences des acheteurs en termes de qualité sont basées sur les indices d'acide et de peroxyde du beurre qui permettent de définir l'utilisation qu'ils peuvent en faire (Lovett, 2006). Ces indices dépendent étroitement du traitement des noix voire des amandes, d'où une maîtrise des procédés d'extraction s'avère nécessaire.

A cet effet, la maîtrise des procédés d'extraction, la caractérisation des beurres extraits et la définition de leurs qualités et de leur fonctionnalité, ont suscité un intérêt au vu des travaux scientifiques et techniques dans certains pays comme le Burkina Faso, le Cameroun (Womeni, 2004 ; NKouam, 2007). Au Bénin, par contre, très peu d'études ont été réalisées dans ce sens. Par conséquent, l'étude des procédés traditionnels d'extraction du beurre de karité produit au Bénin devient un impératif pour répondre aux exigences des marchés aussi bien régionaux qu'internationaux. En effet, il existe diverses méthodes d'extraction du beurre de karité qui varient d'une région à une autre et d'une tribu à une autre (Kassamba, 1997; N'Gouro 1997). Au Bénin par exemple, les procédés d'extraction du beurre de karité communément pratiqués sont ceux des populations du nord: les procédés Lokpa et Peulh. Ces procédés ne sont rien d'autres que des suites d'opérations unitaires mécaniques (dépulpage manuel, lavage, décorticage, pilage, mouture, barattage) et thermiques c'est-à-dire faisant intervenir la chaleur (cuisson, séchage, fumage, torréfaction, chauffage).

Traditionnellement, ces opérations unitaires thermiques se déroulent dans des conditions très peu contrôlées et sont susceptibles de conduire à des modifications biochimiques de natures lipolytique et/ou oxydative, pouvant donc conduire à l'augmentation des indices d'acide et de peroxyde du beurre; ce qui le disqualifie pour certaines destinations. La température et l'apport d'énergie calorifique sont alors deux facteurs très importants dont la maîtrise est indispensable lors des traitements technologiques, car leur influence est prépondérante sur la qualité des produits alimentaires. Cette approche est également valable dans le cadre d'études conceptuelles de procédés améliorés pour la modernisation des méthodes traditionnelles.

La présente étude vise ainsi à déterminer la valeur de la température pour chaque opération thermique impliquée dans les procédés traditionnels d'extraction du beurre de karité au Bénin, et à analyser leurs effets possibles sur la qualité du beurre afin d'envisager les meilleures conditions de traitement.

\section{MATERIEL ET METHODES \\ Matériel}

Les fruits de karité utilisés ont été collectés dans les villages Modji-Gangan, Houéclé et Arigbokoto de la commune de Dassa-Zoumé au Bénin avec une température et une humidité relatives moyennes, respectives de $32 \pm 2{ }^{\circ} \mathrm{C}$ et $70 \pm 10 \%$, au cours des mois de juillet et août.

Tous les solvants utilisés pour les analyses physicochimiques sont de types analytiques et proviennent de la firme Sigma.

Un thermomètre à mercure, un thermocouple à sonde (modèle Cobra 4) et un thermo-hygromètre de marque «Testo» sont utilisés pour les mesures de températures des différentes opérations thermiques, d'hygrométrie relative d'air sur les sites expérimentaux.

\section{Méthodes}

Procédés traditionnels d'extraction du beurre de karité et extraction de beurre

Les procédés d'extraction du beurre de karité mis en jeu au cours de cette étude sont 
ceux observés dans les zones productrices de beurre de karité de la commune de DassaZoumè. Ces procédés sont: le procédé Lokpa, le procédé Peulh et le procédé par fumage. Ces différents procédés sont comparés à une variante du procédé Tchabal pratiqué au cameroun (Womeni, 2004 ; Nkouam, 2007).

Dans le procédé Lokpa décrit dans le diagramme de la Figure 1A, les fruits collectés sont dépulpés et les noix obtenues sont bouillies pendant 75 minutes. Elles sont ensuite refroidies et étalées au soleil en monocouche sur une nappe en polystyrène noir pendant 6 jours. Après décorticage, les amandes sont séchées à nouveau au soleil pendant 15 jours, afin d'être concassées, torréfiées et broyées. La pâte obtenue est ensuite diluée dans de l'eau tiède et brassée d'un mouvement régulier jusqu'à l'obtention des matières grasses qui sont recueillies et lavées à l'eau froide. La crème blanche obtenue est chauffée pour débarrasser le beurre des impuretés.

Le procédé Peulh présenté sur la Figure $1 \mathrm{~B}$, diffère du procédé Lokpa par l'opération de friture qui remplace les opérations de concassage et de torréfaction des amandes. Elle permet ainsi d'éviter la difficile et longue opération de concassage. Un kilogramme de beurre précédemment produit permet de frire $6,25 \mathrm{~kg}$ d'amande.

Le procédé par fumage (Figure 2C) diffère du procédé Lokpa par le fumage successif des noix et des amandes dans un fumoir traditionnel.

Dans le procédé Tchabal (Figure 2D), les noix de karité sont cuites pendant 2 heures puis décortiquées, pilées et laminées afin d'obtenir le beurre. Les beurres obtenus de ces différents procédés sont comparés entre eux, de même qu'avec des beurres extraits au soxhlet de prélèvement de noix ou d'amandes, effectués en amont et en aval de chaque opération thermique dans les différents procédés utilisés.

Ainsi, les prélèvements des fruits, des noix cuites, des noix cuites séchées, des amandes séchées, des amandes séchées et torréfiées, des amandes séchées et frites, des amandes fumées ont été étudiés.

\section{Analyses physico-chimiques}

Elles ont consisté à la détermination de la teneur en eau, de l'indice de réfraction, de l'indice d'acide, de peroxyde et de la composition en acides gras. La teneur en eau et matières volatiles, l'acidité, les indices de réfraction, de peroxyde et de saponification des beurres ont été respectivement déterminés suivant les normes NF 60-201, NF 60-204, NF 60-212, NF T 60-220

La détermination de la composition en acide gras a été faite selon la méthode décrite par Tchobo et al. (2007). Les esters méthyliques des échantillons sont préparés par trans-estérification avec du méthylate de sodium selon la norme NF T60-233. Dans un ballon de $25 \mathrm{~mL}$, on introduit $10 \mathrm{mg}$ de matières grasses auxquels sont ajoutés $3 \mathrm{~mL}$ de solution de méthylate de sodium contenant la phénolphtaléine. Le mélange réactionnel est chauffé à reflux pendant 10 minutes. Puis on ajoute $3 \mathrm{~mL}$ d'une solution méthanolique de $\mathrm{HCl}$ jusqu'à la décoloration de phénolphtaléine. Le mélange ainsi obtenu est à nouveau chauffé à reflux pendant 10 minutes et refroidi à température ambiante. Après ajout de $8 \mathrm{~mL}$ d'hexane et de $10 \mathrm{~mL}$ d'eau, la phase organique contenant les esters méthyliques est récupérée et ensuite séchée avec le sulfate de sodium anhydre. Les esters méthyliques ainsi préparés ont été ensuite analysés au moyen d'un chromatographe Agilent 6890 series équipé d'une colonne capillaire supelcowax $10\{\mathrm{~L}=30 \mathrm{~m} ; \mathrm{D}=320$ $\mu \mathrm{m} ; \mathrm{e}=0,25 \mu \mathrm{m})\}$, d'un injecteur split (rapport de fuite : 1/80) et d'un détecteur à ionisation de flamme. Les températures du détecteur et de l'injecteur étaient respectivement de 250 et $270{ }^{\circ} \mathrm{C}$ et le four a été programmé de $150{ }^{\circ} \mathrm{C}$ à $225^{\circ} \mathrm{C}$ avec un gradient de $5{ }^{\circ} \mathrm{C} /$ minute. Le gaz vecteur est de l'Hélium avec un débit de $1 \mathrm{~mL} /$ minute. L'identification des esters méthyliques a été faite par comparaison des temps de rétention avec les esters méthyliques de témoins préalablement analysés. 

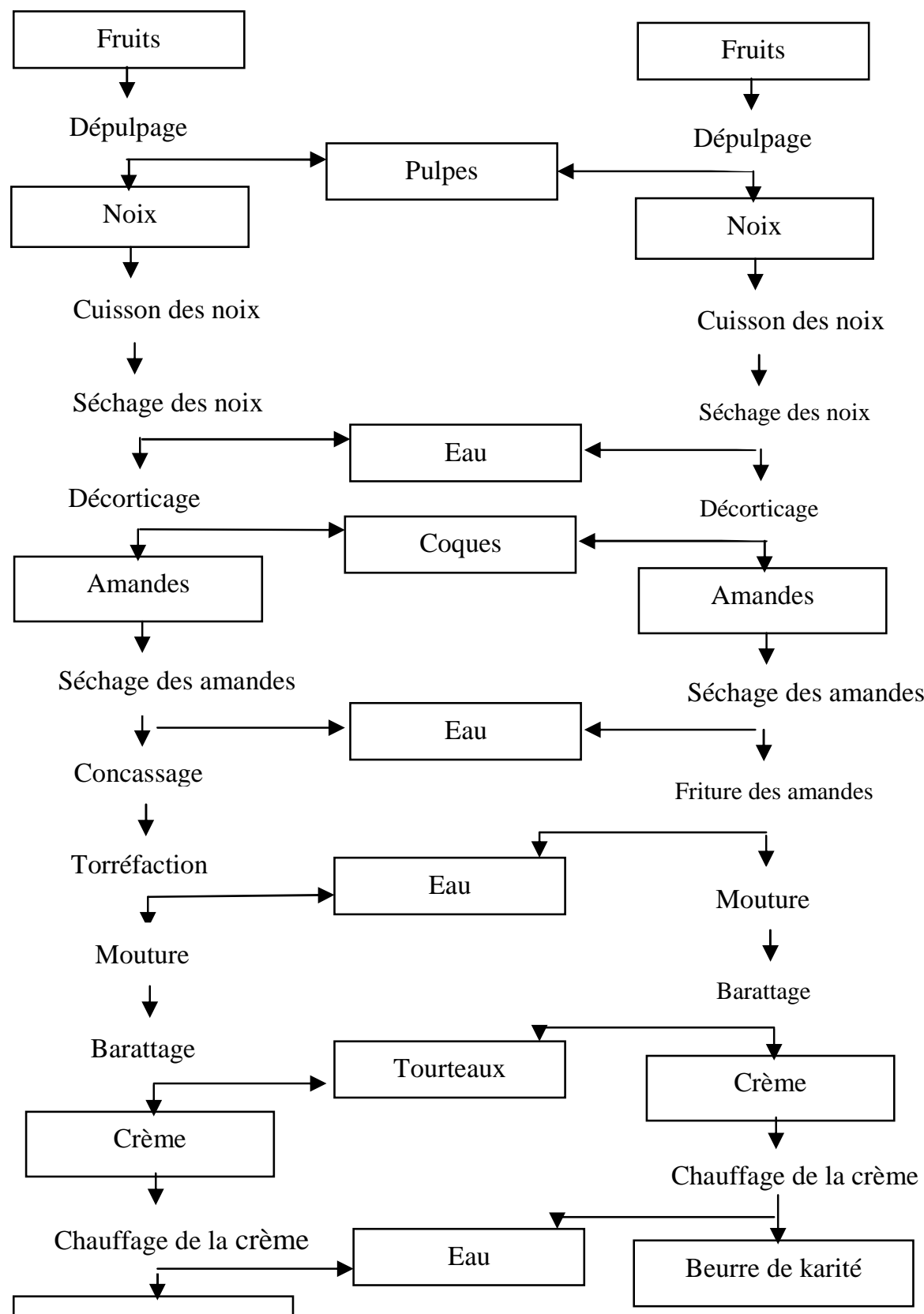

Beurre de karité

(B)

(A)

Figure 1 : Procédé traditionnel d'extraction mis en œuvre - (A): Procédé traditionnel Lokpa, (B): Procédé traditionnel Peulh. 

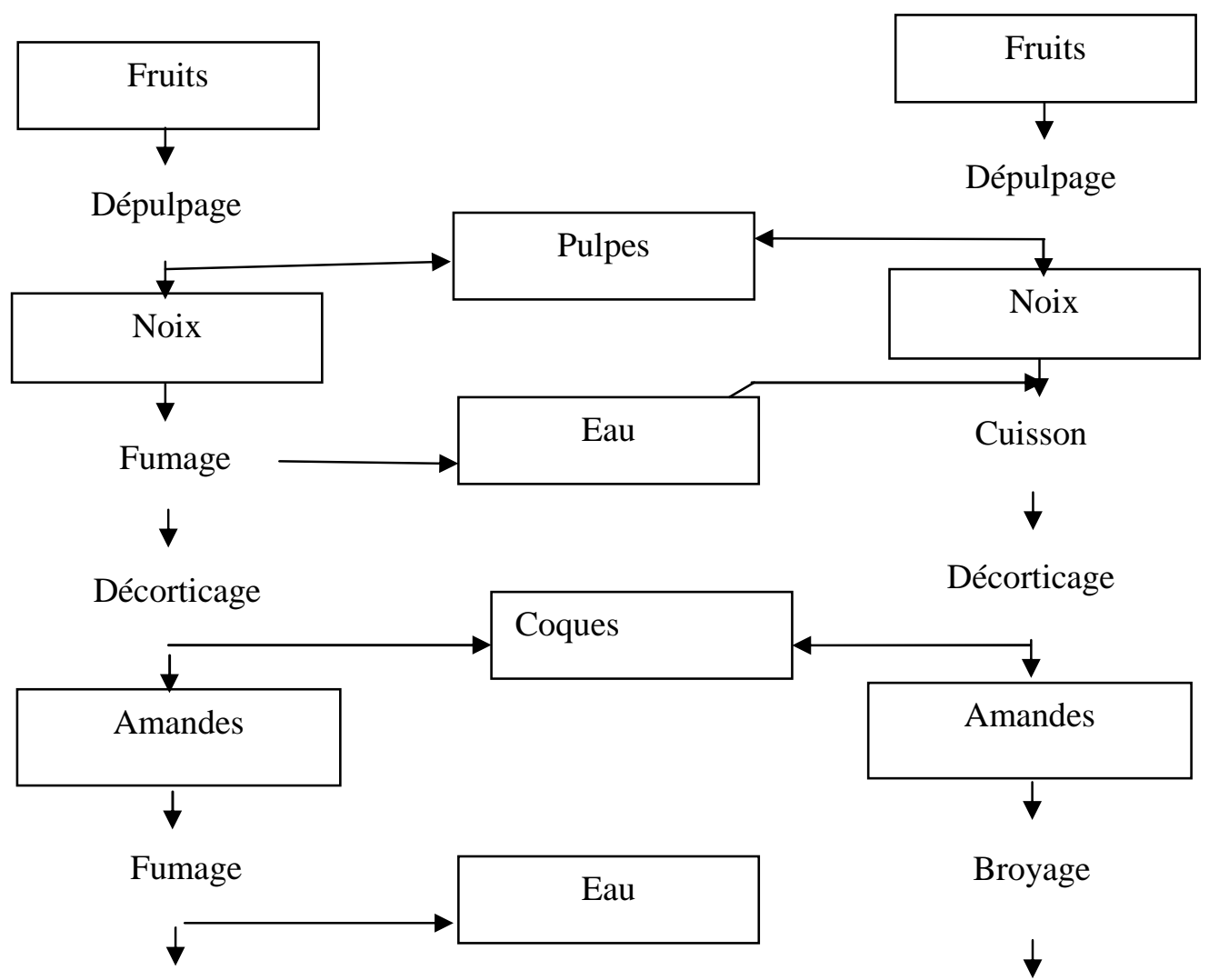

Décorticage

Mouture
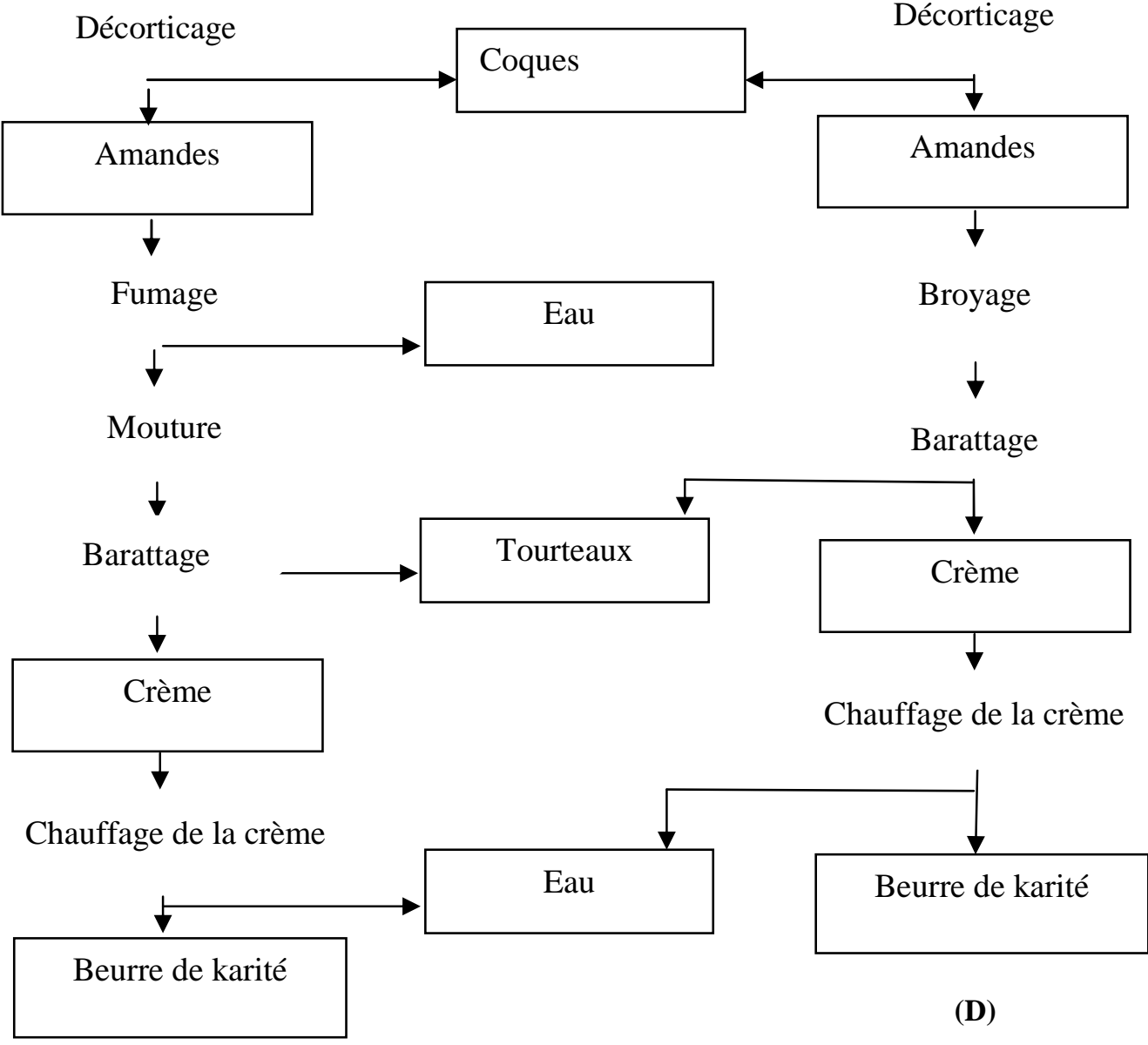

(C)

Figure 2: Procédé traditionnel d'extraction; (C): Procédé traditionnel par fumage ; (D) : Procédé traditionnel Tchabal. 


\section{Analyse statistique}

Toutes les données des analyses physico-chimiques ont été traitées à l'aide des logiciels Microsoft Excel 2007 et SPSS v 16.0. Le test de Student Newman Keuls a été utilisé pour la comparaison des moyennes et l'analyse de la variance.

\section{RESULTATS}

Lors de la production du beurre de karité, les températures des différentes opérations thermiques au niveau des différents procédés étudiés sont regroupées dans le Tableau 1. Les températures enregistrées varient entre 105 et $216{ }^{\circ} \mathrm{C}$ au cours des opérations de cuisson, de friture, de fumage et chauffage de la crème, tandis qu'au cœur de l'amande, elles fluctuent entre 93 et 133 ${ }^{\circ} \mathrm{C}$.

Les Tableaux 2, 3, 4 et 5 donnent pour chaque procédé, les valeurs de la teneur en eau, de l'indice d'acide, de peroxyde et de réfraction des beurres. Ces valeurs, qui portent des lettres différentes sur la même ligne dans les tableaux, sont significativement différentes au seuil de $5 \%$. Dans les différents procédés, on constate une baisse de l'indice d'acide dans le beurre final. Quant à l'indice de peroxyde, il connait une augmentation dans le produit final après l'application de tous les procédés sauf dans le procédé de fumage. La Figure 3 présente l'évolution des indices de qualité du beurre en fonction du temps de cuisson. Enfin, les compositions en acide gras des différents échantillons de beurre obtenus à partir des différents prélèvements des procédés étudiés sont listées dans les Tableaux 6, 7, 8 et 9 .

\section{DISCUSSION}

Les différents paramètres étudiés au cours des différentes opérations thermiques présentent une variation en fonction de l'opération considérée. Ainsi, les réductions de l'acidité observées dans les procédés étudiés au cours de la cuisson et du fumage seraient dues à l'inactivation des enzymes par blocage de la germination des graines. Ces résultats confirment les observations de différents auteurs (Womeni et al., 2003 ; Aissi et al., 2011). En effet, la germination réduit le rendement d'extraction du beurre et lui donne un goût amère (Womeni et al., 2006). De plus, le fumage entraîne la réduction de la teneur en eau, ce qui limiterait l'hydrolyse chimique.

Tableau 1: Valeurs moyennes des températures des opérations thermiques.

\begin{tabular}{|c|c|c|c|}
\hline Procédés & Opérations thermiques & $\begin{array}{l}\text { Température de } \\
\text { l'opération }\left({ }^{\circ} \mathrm{C}\right)\end{array}$ & $\begin{array}{c}\text { Température au cour } \\
\text { de l'amande pendant } \\
\text { l'opération }\left({ }^{\circ} \mathrm{C}\right)\end{array}$ \\
\hline \multirow{4}{*}{ Lokpa } & Cuisson des noix (75 mn) & $105 \pm 0,5$ & $94,5 \pm 0,5$ \\
\hline & $\begin{array}{c}\text { Séchage naturel des noix (6 jrs), } \\
\text { amandes ( } 15 \text { jrs })\end{array}$ & $32 \pm 2$ & - \\
\hline & Torréfaction & $181 \pm 19$ & - \\
\hline & Chauffage de la crème & $104 \pm 3$ & - \\
\hline \multirow{4}{*}{ Peulh } & Cuisson des noix & $105 \pm 0,5$ & $94,5 \pm 0,5$ \\
\hline & $\begin{array}{c}\text { Séchage naturel des noix (6 jrs), } \\
\text { amandes (15 jrs) }\end{array}$ & $32 \pm 2$ & - \\
\hline & Friture des amandes & $171 \pm 9$ & $133 \pm 4$ \\
\hline & Chauffage de la crème & $108 \pm 5$ & - \\
\hline \multirow{2}{*}{$\begin{array}{l}\text { Procédé par } \\
\text { fumage }\end{array}$} & $\begin{array}{l}\text { Fumage des noix }(6 \mathrm{~h}), \\
\text { amandes }(22 \mathrm{~h})\end{array}$ & $158 \pm 44$ & $93 \pm 9$ \\
\hline & Chauffage de la crème & $195 \pm 35$ & - \\
\hline \multirow{2}{*}{ Tchabal } & Cuisson des noix $(2 \mathrm{~h})$ & $105 \pm 0,5$ & $94,5 \pm 0,5$ \\
\hline & Chauffage de la crème $(2 \mathrm{~h})$ & $145 \pm 20$ & - \\
\hline
\end{tabular}




\section{AHOUANNOU et al. / Int. J. Biol. Chem. Sci. 7(5): 2151-2164, 2013}

Tableau 2 : Teneur en eau, indices d'acide, de peroxyde et de réfraction des beurres (extraits à l'hexane) par le procédé Lokpa.

\begin{tabular}{|c|c|c|c|c|c|c|}
\hline \multirow[b]{2}{*}{ Paramètres } & \multicolumn{5}{|c|}{ Beurre } & \multirow{2}{*}{$\begin{array}{c}\text { Beurre du } \\
\text { Procédé Lokpa } \\
\text { (Bénin) }\end{array}$} \\
\hline & Fruits & Noix cuites & $\begin{array}{c}\text { Noix cuites } \\
\text { séchées }\end{array}$ & $\begin{array}{c}\text { Amandes } \\
\text { séchées }\end{array}$ & $\begin{array}{c}\text { Amandes } \\
\text { séchées torréfiées }\end{array}$ & \\
\hline Température $\left({ }^{\circ} \mathrm{C}\right)$ & - & $105 \pm 0,5$ & $32 \pm 2$ & $32 \pm 2$ & $181 \pm 19$ & - \\
\hline Teneur en eau $(\%)$ & $2,51 \pm 0,4$ & $4,3 \pm 0,21$ & $5,9 \pm 0,71$ & $1,24 \pm 0,1$ & $0,98 \pm 0,02$ & $0,33 \pm 0,005$ \\
\hline Indice d'acide (\%) & $15,72 \pm 0,77^{\mathrm{a}}$ & $1,08 \pm 0,03^{\mathrm{b}}$ & $1,66 \pm 0,01^{\mathrm{b}}$ & $1,65 \pm 0,02^{\mathrm{b}}$ & $1,085 \pm 0,18^{\mathrm{b}}$ & $0,97 \pm 0,18^{\mathrm{b}}$ \\
\hline Indice de peroxyde (méq /kg) & $1,59 \pm 0,29^{a}$ & $4,66 \pm 0,65^{\mathrm{b}}$ & $1,22 \pm 0,23^{\mathrm{c}}$ & $3,25 \pm 1,05^{\mathrm{d}}$ & $1 \pm 0,47^{\mathrm{e}}$ & $7,06 \pm 0,87^{f}$ \\
\hline Indice de réfraction & $1,4695^{\mathrm{a}}$ & $1,4730^{\mathrm{b}}$ & $1,4682^{c}$ & $1,4727^{\mathrm{d}}$ & $1,4682^{\mathrm{e}}$ & $1,4680^{\mathrm{e}}$ \\
\hline
\end{tabular}

Les valeurs portant la même lettre dans la même ligne ne sont pas significativement différentes $(\mathrm{p}<0,05)$.

Tableau 3: Teneur en eau, indices d'acide, de peroxyde et de réfraction des beurres (extraits à l'hexane) des échantillons du procédé Peulh.

\begin{tabular}{|c|c|c|c|c|c|c|}
\hline \multirow[b]{2}{*}{ Paramètres } & \multicolumn{5}{|c|}{ Beurre } & \multirow{2}{*}{$\begin{array}{l}\text { Beurre du Procédé } \\
\text { Peulh (Bénin) }\end{array}$} \\
\hline & Fruits & Noix cuites & $\begin{array}{l}\text { Noix cuites } \\
\text { séchées }\end{array}$ & $\begin{array}{l}\text { Amandes } \\
\text { séchées }\end{array}$ & $\begin{array}{c}\text { Amandes séchées } \\
\text { frites }\end{array}$ & \\
\hline Température $\left({ }^{\circ} \mathrm{C}\right)$ & - & $105 \pm 0,5$ & $32 \pm 2$ & $32 \pm 2$ & $171 \pm 9$ & - \\
\hline Teneur en eau (\%) & $2,51 \pm 0,4$ & $4,3 \pm 0,21$ & $5,9 \pm 0,71$ & $1,24 \pm 0,1$ & $0,71 \pm 0,001$ & $0,09 \pm 0,001$ \\
\hline Indice d'acide (\%) & $15,72 \pm 0,77^{\mathrm{a}}$ & $1,08 \pm 0,03^{\mathrm{b}}$ & $1,66 \pm 0,01^{\mathrm{b}}$ & $1,65 \pm 0,02^{\mathrm{b}}$ & $0,89 \pm 0,20^{\mathrm{b}}$ & $0,97 \pm 0,19^{\mathrm{b}}$ \\
\hline Indice de peroxyde (méq/kg) & $1,59 \pm 0,29^{\mathrm{a}}$ & $4,66 \pm 0,65^{\mathrm{b}}$ & $1,22 \pm 0,23^{\mathrm{c}}$ & $3,25 \pm 1,05^{\mathrm{d}}$ & $2,12 \pm 0,29^{\mathrm{e}}$ & $6,72 \pm 0,66^{\mathrm{f}}$ \\
\hline Indice de réfraction & $1,4695^{\mathrm{a}}$ & $1,4730^{\mathrm{b}}$ & $1,4682^{c}$ & $1,4727^{\mathrm{d}}$ & $1,4702^{\mathrm{e}}$ & $1,4682^{\mathrm{f}}$ \\
\hline
\end{tabular}


Tableau 4: Teneur en eau, indices d'acide, de peroxyde et de réfraction des beurres (extraits à l'hexane) des échantillons du procédé de Tchabal.

\begin{tabular}{lccc}
\hline \multirow{2}{*}{ Paramètres } & \multicolumn{2}{c}{ Beurre } & $\begin{array}{c}\text { Beurre du procédé } \\
\text { Tchabal (Cameroun) }\end{array}$ \\
\cline { 2 - 3 } & Fruits & Noix cuites & - \\
Température $\left({ }^{\circ} \mathrm{C}\right)$ & - & $105 \pm 0,5$ & - \\
Teneur en eau $(\%)$ & $4,22 \pm 0,57$ & $4,3 \pm 0,49$ & $0,53 \pm 0,05$ \\
Indice d'acide $(\%)$ & $10,31 \pm 0,12 \mathrm{a}$ & $1,03 \pm 0,09 \mathrm{~b}$ & $1,31 \pm 0,06 \mathrm{~b}$ \\
Indice de peroxyde $(\mathrm{méq} / \mathrm{kg})$ & $1,32 \pm 0,46 \mathrm{a}$ & $10,69 \pm 0,55 \mathrm{~b}$ & $2,76 \pm 0,32 \mathrm{c}$ \\
Indice de réfraction & $1,4677 \mathrm{a}$ & $1,4680 \mathrm{a}$ & $1,4705 \mathrm{~b}$ \\
\hline
\end{tabular}

Les valeurs portant la même lettre dans la même ligne ne sont pas significativement différentes $(\mathrm{p}>0,05)$.

Tableau 5 : Teneur en eau, indices d'acide, de peroxyde et de réfraction des beurres (extraits à l'hexane) des échantillons du procédé par fumage.

\begin{tabular}{lccc}
\hline Paramètres & \multicolumn{2}{c}{ Beurre } & Beurre du procédé \\
\cline { 2 - 3 } & pruits & Amandes fumées & \\
\cline { 2 - 3 } Température $\left({ }^{\circ} \mathrm{C}\right)$ & - & $158 \pm 44$ & - \\
Teneur en eau $(\%)$ & $2,53 \pm 0,32$ & $1,08 \pm 0,07$ & $0,02 \pm 0,001$ \\
Indice d'acide $(\%)$ & $19,19 \pm 0,55 \mathrm{a}$ & $1,55 \pm 0,06 \mathrm{~b}$ & $3,03 \pm 0,14 \mathrm{~b}$ \\
Indice de peroxyde $(\mathrm{méq} / \mathrm{kg})$ & $67,55 \pm 2,55 \mathrm{a}$ & $58,24 \pm 1,70 \mathrm{~b}$ & $29,86 \pm 2,35 \mathrm{c}$ \\
Indice de réfraction & $1,4852 \mathrm{a}$ & $1,4710 \mathrm{~b}$ & $1,4700 \mathrm{~b}$ \\
\hline \multicolumn{2}{c}{ Les valeurs portant la même lettre dans la même ligne ne sont pas significativement différentes $(\mathrm{p}>0,05)}$.
\end{tabular}

Les valeurs portant la même lettre dans la même ligne ne sont pas significativement différentes $(p>0,05)$.

Tableau 6 : composition chimique des échantillons de beurre du procédé Lokpa.

\begin{tabular}{|c|c|c|c|c|c|c|}
\hline \multirow{2}{*}{$\begin{array}{l}\text { Concentration en Acides } \\
\text { Gras }(\%)\end{array}$} & \multicolumn{5}{|c|}{ Beurre } & \multirow{2}{*}{$\begin{array}{l}\text { Beurre du } \\
\text { procédé }\end{array}$} \\
\hline & Fruits & $\begin{array}{l}\text { Noix } \\
\text { cuites }\end{array}$ & $\begin{array}{l}\text { Noix cuites } \\
\text { séchées }\end{array}$ & $\begin{array}{l}\text { Amandes } \\
\text { séchées }\end{array}$ & $\begin{array}{l}\text { Amandes } \\
\text { torréfiées }\end{array}$ & \\
\hline Acide myristique (C14:0) & 0,2 & 0,1 & & & 0,2 & 0,2 \\
\hline Acide palmitique (C16:0) & 4,5 & 4,2 & 4,3 & 4,4 & 4,6 & 4,4 \\
\hline Acide palmitoléique (C16:1) & 0,1 & & & & & 0,1 \\
\hline Acide stéarique (C18:0) & 39,2 & 38,9 & 38,9 & 40,8 & 40,2 & 39,3 \\
\hline Acide oléique (C18:1) & 46,4 & 47,7 & 46,7 & 45,2 & 45,3 & 46,7 \\
\hline Acide linoléique (C18:2) & 7,9 & 7,5 & 8,2 & 7,1 & 8,1 & 7,5 \\
\hline Acide linolénique (C18:3) & 0,2 & 0,2 & 0,2 & 1,2 & 0,2 & 0,2 \\
\hline Acide arachidique (C20:0) & 1,2 & 1,2 & 1,2 & 1,1 & 1,1 & 1,2 \\
\hline Acide éicosénoïque (C20:1) & 0,3 & 0,3 & 0,3 & 0,2 & 0,3 & 0,3 \\
\hline Acides gras saturés & 45,1 & 44,4 & 44,4 & 46,3 & 46,2 & 45,0 \\
\hline Acides gras monoinsaturés & 46,8 & 47,9 & 46,9 & 45,4 & 45,5 & 47,1 \\
\hline Acides gras polyinsaturés & 8,1 & 7,7 & 8,4 & 8,3 & 8,3 & 7,8 \\
\hline
\end{tabular}


Tableau 7 : Composition chimique des échantillons de beurre du procédé Peulh.

\begin{tabular}{|c|c|c|c|c|c|c|}
\hline \multirow[b]{2}{*}{$\begin{array}{l}\text { Concentration en Acide } \\
\text { Gras }(\%)\end{array}$} & \multicolumn{5}{|c|}{ Beurre } & \multirow[b]{2}{*}{$\begin{array}{c}\text { Beurre } \\
\text { du } \\
\text { procédé }\end{array}$} \\
\hline & Fruits & $\begin{array}{l}\text { Noix } \\
\text { cuites }\end{array}$ & $\begin{array}{c}\text { Noix } \\
\text { cuites } \\
\text { séchées }\end{array}$ & $\begin{array}{l}\text { Amandes } \\
\text { séchées }\end{array}$ & $\begin{array}{l}\text { amandes } \\
\text { frites }\end{array}$ & \\
\hline Acide myristique (C14:0) & 0,2 & 0,1 & & & 0,2 & 0,0 \\
\hline Acide palmitique (C16:0) & 4,5 & 4,2 & 4,3 & 4,4 & 4,0 & 4,1 \\
\hline Acide palmitoléique (C16:1) & 0,1 & & & & 0,1 & 0,1 \\
\hline Acide stéarique (C18:0) & 39,2 & 38,9 & 38,9 & 40,8 & 40,3 & 42,0 \\
\hline Acide oléique (C18:1) & 46,4 & 47,7 & 46,7 & 45,2 & 45,7 & 45,5 \\
\hline Acide linoléique (C18:2) & 7,9 & 7,5 & 8,2 & 7,1 & 7,9 & 6,5 \\
\hline Acide linolénique (C18:3) & 0,2 & 0,2 & 0,2 & 1,2 & 0,2 & 0,2 \\
\hline Acide arachidique (C20:0) & 1,2 & 1,2 & 1,2 & 1,1 & 1,2 & 1,3 \\
\hline Acide éicosénoïque (C20:1) & 0,3 & 0,3 & 0,3 & 0,2 & 0,3 & 0,3 \\
\hline Acides gras saturés & 45,1 & 44,4 & 44,4 & 46,3 & 45,8 & 47,4 \\
\hline Acides gras monoinsaturés & 46,8 & 47,9 & 46,9 & 45,4 & 46,1 & 45,9 \\
\hline Acides gras polyinsaturés & 8,1 & 7,7 & 8,4 & 8,3 & 8,1 & 6,7 \\
\hline
\end{tabular}

Tableau 8 : Composition chimique des échantillons de beurre du procédé par fumage.

\begin{tabular}{|c|c|c|c|}
\hline \multicolumn{3}{|c|}{ Beurre } & \multirow[b]{2}{*}{ Beurre après Fumage } \\
\hline $\begin{array}{l}\text { Concentration en Acide } \\
\text { Gras }(\%)\end{array}$ & Fruit & Amandes fumées & \\
\hline Acide myristique (C14:0) & 0,2 & 0,3 & - \\
\hline Acide palmitique (C16:0) & 4,7 & 4,7 & 4,4 \\
\hline Acide stéarique (C18:0) & 41,0 & 41,8 & 46,8 \\
\hline Acide oléique (C18:1) & 44,9 & 43,9 & 41,1 \\
\hline Acide linoléique( C18:2) & 7,7 & 7,8 & 6,6 \\
\hline Acide linolénique (C18:3) & 0,2 & 0,2 & - \\
\hline Acide arachidique (C20:0) & 1,1 & 1,1 & 1,1 \\
\hline Acide éicosénoïque (C20:1) & 0,2 & 0,1 & - \\
\hline Acides gras saturés & 47,0 & 47,9 & 52,2 \\
\hline Acides gras monoinsaturés & 45,1 & 44,0 & 41,1 \\
\hline Acides gras polyinsaturés & 7,9 & 8,1 & 6,6 \\
\hline
\end{tabular}




\section{AHOUANNOU et al. / Int. J. Biol. Chem. Sci. 7(5): 2151-2164, 2013}

Tableau 9 : Composition chimique des échantillons de beurre du procédé Tchabal.

\begin{tabular}{lccc}
\hline \multicolumn{1}{c}{ Beurre } & & Beurre \\
\cline { 1 - 2 } Concentration en Acide Gras (\%) & Fruit & Noix cuites & ProcédéTchabal \\
\hline Acide myristique( C14:0) & 0,1 & - & 0,1 \\
Acide palmitique (C16:0) & 3,8 & 3,4 & 3,8 \\
Acide palmitoléique (C16:1) & 0,0 & - & 0,1 \\
Acide stéarique (C18:0) & 40,6 & 37,6 & 43,5 \\
Acide oléique (C18:1) & 45,8 & 49,3 & 44,0 \\
Acide linoléique (C18:2) & 6,8 & 7,8 & 6,6 \\
Acide linolénique (C18:3) & 0,2 & 0,2 & 0,2 \\
Acide arachidique (C20:0) & 1,3 & 1,3 & 1,3 \\
Acide éicosénoïque (C20:1) & 0,3 & 0,4 & 0,3 \\
Acides gras saturés & 45,8 & 42,4 & 48,7 \\
Acides gras monoinsaturés & 46,1 & 49,7 & 44,4 \\
Acides gras polyinsaturés & 7,0 & 8,0 & 6,9 \\
\hline
\end{tabular}

Tableau 10 : Evolution de la qualité du beurre en fonction du temps de cuisson des noix.

\begin{tabular}{|c|c|c|c|c|c|c|c|}
\hline \multirow[b]{2}{*}{ Paramètres } & \multicolumn{7}{|c|}{ Beurre } \\
\hline & 00 min & $30 \mathrm{~min}$ & $60 \mathrm{~min}$ & $90 \mathrm{~min}$ & $120 \mathrm{~min}$ & $150 \mathrm{~min}$ & $180 \mathrm{~min}$ \\
\hline Teneur en eau & $3,1 \pm 0,05$ & $2,2 \pm 0,01$ & $2,5 \pm 0,04$ & $1,9 \pm 0,02$ & $2,0 \pm 0,01$ & $2,8 \pm 0,02$ & $2,7 \pm 0,01$ \\
\hline Indice d'acide & $10,22 \pm 0,54^{\mathrm{a}}$ & $3,13 \pm 0,27^{\mathrm{b}}$ & $8,09 \pm 0,26^{\mathrm{c}}$ & $5,43 \pm 0,04^{\mathrm{d}}$ & $11,49 \pm 0,06^{\mathrm{e}}$ & $4,70 \pm 0,32^{\mathrm{f}}$ & $8,64 \pm 0,25^{\mathrm{g}}$ \\
\hline Indice de peroxyde & $1,98 \pm 0,46^{\mathrm{a}}$ & $6,53 \pm 1,00^{\mathrm{b}}$ & $8,46 \pm 0,08^{\mathrm{c}}$ & $10,38 \pm 0,54^{\mathrm{d}}$ & $12,64 \pm 0,09^{\mathrm{e}}$ & $13,01 \pm 0,54^{\mathrm{e}}$ & $14,72 \pm 0,12^{\mathrm{f}}$ \\
\hline Indice de réfraction & 1,46775 & 1,46875 & 1,46875 & 1,4695 & 1,4680 & 1,46925 & 1,4690 \\
\hline
\end{tabular}

Les valeurs portant la même lettre dans la même ligne ne sont pas significativement différents $(\mathrm{p}>0,05)$. 


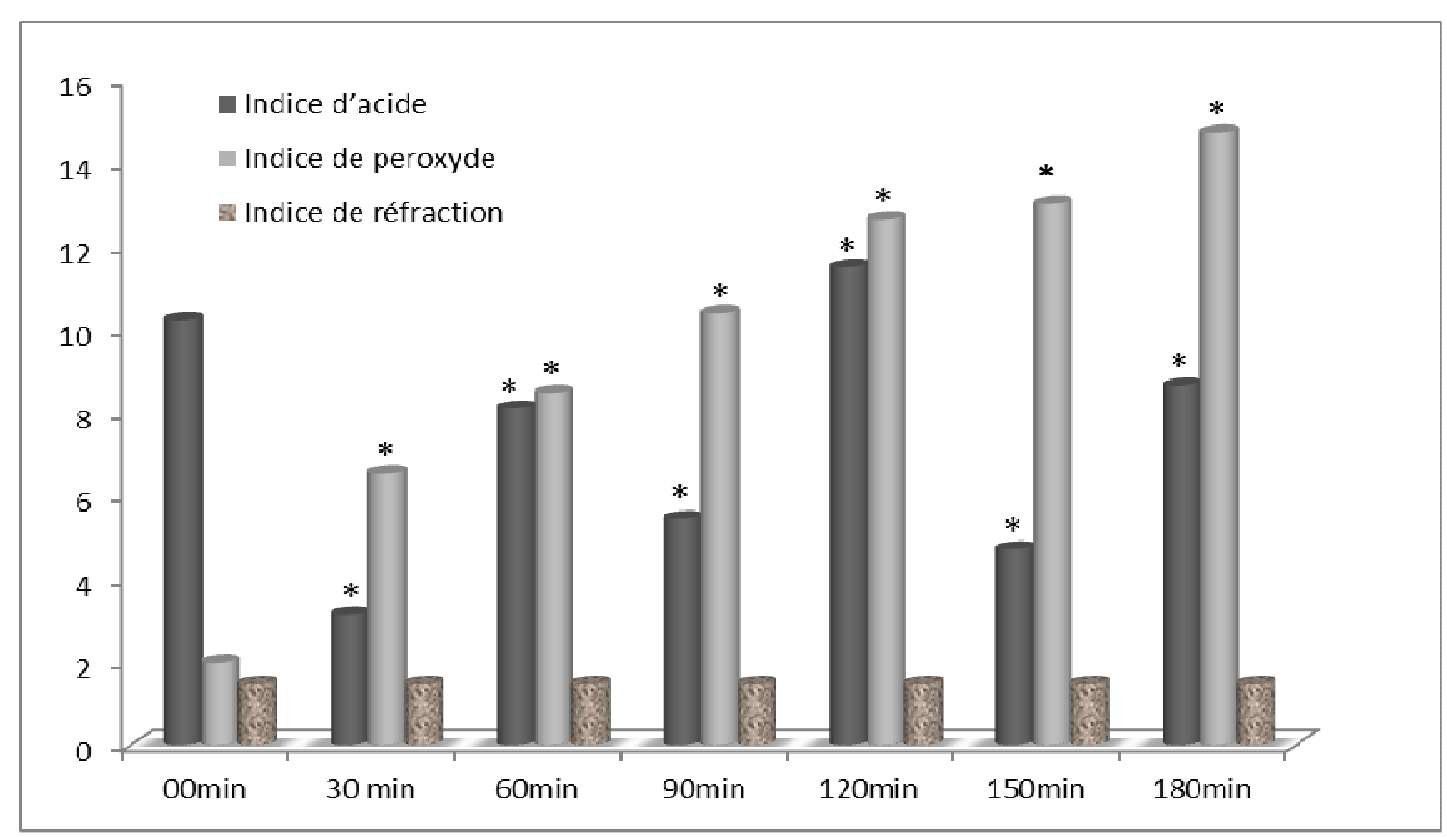

Figure 3: Evolution de la qualité du beurre en fonction du temps de cuisson des noix. Avec des différences significatives sur l'histogramme présentant un seuil de probabilité de $5 \%$, soit $\mathrm{P}<0,05$.

Cependant, si la cuisson augmente les risques d'hydrolyse chimique des triacylglycerols par l'action simultanée de la température et de l'eau, la baisse de l'acidité des beurres constatée peut aussi s'expliquer par le lessivage des acides gras (dissolution des acides gras) par l'eau de cuisson. Ce phénomène de dissolution a été évoqué par Aissi et al. (2011) dans l'étude de l'extraction du beurre de Pentadesma. Quant à l'indice de peroxyde qui augmente au cours de la cuisson des noix $(1,59$ à 4,66 méq/kg, 1,59 à 4,66 méq/kg, 1,32 à 10,69 méq/kg, respectivement pour les procédés Lokpa, Peulh et Tchabal), elle peut s'expliquer par le fait que la température élevée de la cuisson aurait non seulement inactivé les enzymes responsables de l'oxydation enzymatique, mais elle aurait, avec le concours d'une humidité élevée, catalysé une oxydation non enzymatique, d'où la production des peroxydes (Womeni et al., 2003 ; Aissi et al., 2011). La Figure 3, qui montre l'augmentation de la l'indice de peroxyde avec le temps de cuisson, vient de plus renforcer l'hypothèse ci-dessus évoquée. Ainsi, un court temps de cuisson des amandes permettrait d'inactiver les enzymes et limiter la formation des peroxydes pour une amélioration de la qualité du beurre. Toutefois, selon Womeni et al. (2006), des temps de cuisson inférieurs à 50 minutes pour inactiver les lipases ne seraient que théoriques et seraient insuffisants pour que la chaleur atteigne le cour des amandes de grandes épaisseurs. Cependant, cette étude a montré qu'avec une cuisson dans un bain d'eau à température constante, il s'en suit une très bonne répartition de la chaleur qui infuse de part et d'autre les noix. En effet, la température au cœur de l'amande mesurée après 10 minutes de cuisson est de $94,5^{\circ} \mathrm{C}$ en dépit de l'épaisseur de la noix. Une prolongation du temps de cuisson 
augmenterait inutilement le coût de l'énergie utilisée.

Le beurre final obtenu à partir des procédés Lokpa et Peulh présente aussi des indices de peroxyde élevés par rapport aux beurres issus des amandes de départ et cuites. En effet, selon Aissi et al. (2011), l'augmentation de la surface de contact entre l'oxygène, les acides gras et l'humidité par la mouture est la source de production des peroxydes. Dans le procédé de fumage, les conditions opératoires permettent de réduire l'oxydation du beurre. Ces observations ont été aussi confirmées par les travaux de Aissi et al. (2011). Ce constat peut s'expliquer par le fait que la température du fumage proche de celle de la désodorisation des corps gras, provoque la sortie de l'eau des amandes sous forme de vapeur favorisant l'élimination des peroxydes par entraînement (Pages et al., 2010).

En ce qui concerne les autres opérations unitaires, l'application du séchage solaire fait observer une légère augmentation de l'acidité du beurre dans le cas des noix cuites. En effet, les enzymes étant inactivées par la cuisson, la faible augmentation de l'acidité serait due soit à la colonisation des moisissures colonisatrices pendant le séchage, soit à une hydrolyse chimique. Ces justifications sont confirmées par les travaux de Womeni et al. (2006 et 2007), qui en étudiant l'impact du séchage traditionnel solaire des amandes issues de noix non cuites, ont observé l'acidité du beurre doublée de 32,5 à 63,3\%. Pour la réduction de l'indice de peroxyde observé; elle serait due à la compétition entre la formation et la décomposition des peroxydes suivant le modèle de réactions proposé par Graille (2003). Quant à la torréfaction et la friture, elles ont permis la baisse de l'oxydation. Selon Pages et al. (2010), cette baisse est liée aux températures élevées qui éliminent systématiquement les peroxydes. De plus, la réaction de décomposition prendrait ainsi le pas sur la réaction de formation des peroxydes. Au cours du chauffage de la crème et de l'obtention du beurre à la fin de chaque procédé, on constate une constance dans l'acidité du beurre quand on passe d'un procédé à un autre. Seul le beurre obtenu du fumage a présenté une légère augmentation de son acidité.

En somme, on peut affirmer que pendant les opérations thermiques, il existe une plage de températures qui favorisent la réduction ou l'élimination des peroxydes et une plage de températures qui favorisent l'augmentation ou la formation des peroxydes. Quant à l'acidité, il faut noter une diminution à la fin du processus de transformation.

Les Tableaux 6, 7, 8 et 9 présentent la composition en acides gras des différents échantillons de beurre extrait des fruits de karité collectés. L'analyse de ces Tableaux fait ressortir que les échantillons des procédés Lokpa, Peulh et Tchabal, puis celui de fumage ont une même composition qualitative en acide gras. Dans l'ensemble, on remarque que le profil en acide gras est semblable au profil rencontré dans la littérature (Maranz et al., 2004 ; Kapseu et al., 1998). En effet, le beurre de karité est caractérisé par 4 acides gras que sont l'acide palmitique $(\mathrm{C} 16: 0)$ et l'acide stéarique (C18:0) qui constituent l'essentiel des acides gras saturés; l'acide oléique (C18:1) pour les acides gras monoinsaturés et l'acide linoléique (C18:2) pour les acides gras polyinsaturés. L'analyse de ces résultats fait ressortir également que les traitements thermiques impliqués dans les procédés traditionnels d'extraction du beurre de karité n'affectent ni la composition, ni la teneur des acides gras. En effet, les acides gras du beurre de karité sont thermiquement stables. De plus, une variation du profil en acide gras est souvent liée aux facteurs environnementaux 
tels que les précipitations, la fertilité des sols, la période de maturation, les pratiques agronomiques et la substitution génétique (Maranz et al., 2004)

\section{Conclusion}

Les résultats de cette étude montrent que lors des opérations thermiques impliquées dans les procédés traditionnels d'extraction du beurre de karité, la température n'est pas maîtrisée. Ces opérations sont donc peu contrôlées et sont par principe des sources d'altérations lipolytique et oxydative de la matière grasse. Les indices d'acide et peroxyde du produit final dépendent ainsi des températures mises en œuvre lors des procédés d'extraction. Par contre, la composition en acide gras du beurre n'est pas affectée par la chaleur. Une bonne conduite de ces opérations peut permettre d'obtenir des beurres de premier grade (indice d'acide < $1,1 \%$; indice de peroxyde < $10 \mathrm{méq} / \mathrm{kg}$ ) recherchés en cosmétologie et en pharmacologie, d'après les normes de l'Union Economique Monétaire Ouest Africaine relatives au beurre de karité.

\section{REFERENCES}

Aïssi MV, Tchobo FP, Natta AK, Piombo G, Villeneuve $\mathrm{P}$, Sohounhloué DCK, Soumanou MM. 2011. Effet des prétraitements post-récolte des amandes de Pentadesma butyracea (Sabine) sur la technologie d'extraction en milieu réel et la qualité du beurre. OCL, 18(6): 384392.

APROMA. 1995. Etude de la filière karité du Burkina Faso (Rapport principal). Union Européenne, Délégation de la Commission Européenne, Ouagadougou (BF), p. 15-19.

Compaoré PN. 2000. Femmes, développement et transfert de technologies. Le cas des presses à karité au Burkina Faso. Thèse de doctorat, Université de Montréal, Département de Sociologie, Montréal.

Graille J. 2003. Lipides et Corps Gras Alimentaires. Lavoisier-Tec et Doc: Paris.

Kapseu C. 2009. Production, analyse et applications des huiles végétales en Afrique. OCL., 16(4): 215-221.

Kapseu C, Jiokap NY, Parmentier M, Dirand M, Dellacherie J. 2001. Acides gras et triglycérides du beurre de karité du Cameroun. La Rivista Italiana Delle Sostanze Grasse, LXXVIII: 31-34.

Lovett P. 2006. Spécifications techniques et test au laboratoire pour le beurre de karité et les produits cosmétiques. Karité 2006: Exporting quality and quantity. http://www.watradehub.com/sites/default/ files/resourcefiles/aug09/120la20chaine2 0de20valeur20du20beurre20du20karite20 28j20holtzman29.pdf

Maranz S, Wiesman Z, Bisgaard J, Bianchi G. 2004. Germplasm, resources of Vitellaria paradoxa based on variation in fat composition across the distribution range. Agroforest Syst., 60: 71-76.

N'Gouro S. 1997. Comment avoir du beurre de karité sans transpirer. Le Grenier, 4: 45 .

NKouam GB. 2007. Conservation des fruits du karité (Vitellaria paradoxa Gaertn.) et de l'aiéle (Canarium schweinfurthii Engl.) : isothermes de sorption d'eau et extraction des matières grasses des fruits stockés. Thèse de doctorat Ph.D., Université de Ngaoundéré, (ENSAI), Ngaoundéré (Cameroun), p. 286.

Pages X, Morin O, Birot C, Gaud M, Fazeuilh S, Gouband M. 2010. Raffinage des huiles et des corps gras et élimination des contaminants. OCL, 17(2): 14p.

Tchobo FP, Natta AK, Barea B, Barouh N, Piombo G, Pina M, Villeneuve P, Soumanou MM, Sohounhloue DCK. 
2007. Characterization of Pentadesma butyracea sabine Butters of different production regions in Benin. J. Am. Oil Chem. Soc., 84: 755-760.

Womeni HM. 2004. Identification et analyse des opérations critiques de préparation des fruits, graines et amandes de karité (Butyrospermum parkii (G. Don) Kotschy): étude de leur influence sur la qualité $\mathrm{du}$ beurre. Thèse de Doctorat/Ph.D, Université de Ngaoundéré, Ngaoundéré (Cameroun).

Womeni HM, Ndjouenkeu R, Tchatchueng JB, Kapseu C. 2000. Effet de la conservation des fruits du karité par le froid sur la cinétique de séchage et la qualité de l'huile. Biosciences Proceedings, Mbofung CMF, Etoa F-X (éds), 147-154.

Womeni HM, Kamga R, Tchiégang C, Kapseu C. 2002. Extraction du beurre dekarité : influence du séchage des amandes et de la technique d'extraction, La Rivista Italiana Delle Sostanze Grasse, LXXIX: 33-37
Womeni HM, Tchagna DT, Ndjouenkeu R, Kapseu C, Mbiapo FT, Linder M, Parmentier M, Fanni J. 2003. Influence des traitements traditionnels des graines et amandes de karité sur la qualité du beurre. Food Africa: Improving food systems in sub-Africa, responding to a changing environment, Yaoundé (Cameroun), p. 1-8.

Womeni HM, Ndjouenkeu R, Kapseu C, Tchouanguep MF, Parmentier M, Fanni J. 2007. Séchage des amandes de karité et qualité du beurre: impact du séchage traditionnel au soleil. Tropicultura, 25(4): 240-247.

Womeni HM, Tchagna DT, Ndjouenkeu R, Kapseu C, Mbiapo FT, Linder M, Fanni JJ, Parmentier M. 2006. Effet de la cuisson et du séchage des noix de karité (Butyrospermum parkii (G. Don) Kotschy) sur la qualité du beurre. Tropicultura, 24(3): 175-182. 